\title{
On the shelf resonances of the English Channel and Irish Sea
}

\author{
D. J. Webb \\ National Oceanography Centre, Southampton SO14 3ZH, UK \\ Correspondence to: D. J. Webb (djw@soton.ac.uk) \\ Received: 6 December 2012 - Published in Ocean Sci. Discuss.: 22 February 2013 \\ Revised: 27 June 2013 - Accepted: 12 July 2013 - Published: 26 August 2013
}

\begin{abstract}
The resonances of the English Channel and Irish Sea are investigated using the methods of Webb (2012) together with an Arakawa C-grid model of the region under study. In the semi-diurnal tidal band, the high tides of the Bristol Channel and Gulf of St. Malo are shown to be due to two shelf resonances which strongly couple the two regions. In the diurnal band, the response is complicated by the presence of continental shelf waves.
\end{abstract}

\section{Introduction}

The key role of continental shelf resonances in extracting tidal energy out of the deep ocean was discussed originally by Webb (1976) and later by Buchwald (1980), Huthnance (1980) and Clarke and Battisti (1981). Recent papers include Arbic et al. (2007) and Arbic et al. (2009). Around the British Isles, where a large amount of tidal energy is dissipated (Egbert and Ray, 2000), resonances have been used to explain the high tides of the Bristol Channel (Fong and Heaps, 1978; Heath, 1981).

Grignon (2005) investigated the resonances around the British Isles using a model code developed by Webb (1981) for a study of the Gulf of Carpentaria and the Arafura Sea. It was found that the model generated realistic tides in the North Sea but it failed in the English Channel and Irish Sea. At the time the reason for this was not understood, but it is now realized that although the finite-difference scheme was accurate to second order in the grid spacing, it was not written in an energy-conserving form. The resulting errors appear to have remained small when the model was used with the relatively smooth coastlines of the Gulf of Carpentaria or the North Sea, but were much larger when used for the English Channel and Irish Sea, where the coastline includes more regions with high curvature.
For the present paper the core section of the model code has been rewritten to represent the shallow-water equations in energy-conserving form on an Arakawa C grid (Arakawa, 1966). This is the scheme used by most of the two- and three-dimensional time-dependent models that have been used to study the region (Pingree and Griffiths, 1978; Owen, 1980; Le Provost et al., 1981; Stephens, 1986; Flather, 1987; Kwong et al., 1997; Sinha and Pingree, 1997; Jones and Davies, 2005; Uncles, 2010).

When the present model is forced on the open boundary by the Atlantic semi-diurnal and diurnal tides, the resulting pattern of tides within the region provides a reasonable match to the observed tides. Although the model is still not perfect, the results provide the confidence needed to extend the use of the model to investigate the resonances of the English Channel and Irish Sea. The approach used is similar to that tried by Grignon (2005) and applied successfully by Webb (2012) in a study of the Gulf of Carpentaria.

Before continuing, it needs to be emphasized that the present study and ones like it cannot be more than preliminary studies of the resonances of the ocean. This is because, in reality, the resonances must be modes of the global ocean.

Problems involving global tides can be studied with timestepping models. However, when the underlying equations are converted to the matrix form used here, the size of the matrix and the computation cost of solution requires a major computational effort.

The present preliminary study of European shelf resonances has therefore concentrated on a manageable region of the European shelf itself. By doing this, it implicitly assumes that at a particular real angular velocity, the important resonances of the global ocean will have spatial structures, in the English Channel and Irish Sea, similar to shelf resonances of the present model. This is usually true for physical 
systems, and was found by Arbic et al. (2009) in their study of coupled systems.

Returning to this preliminary study, the "real" response of the model region to forcing by the Atlantic is first investigated by running the model at angular velocity intervals of 0.1 radians per day, between 0 and 30 radians per day. This provides the type of information that can be produced by a time-dependent ocean model forced at different frequencies.

However the present model can be also run with complex values of angular velocity. The response function calculations are therefore extended to cover the rectangular region of the complex plane whose corners are defined by the origin and the points $(0-10 i),(30-10 i)$ and 30 radians per day. The continental shelf resonances now show as analytic poles in the response function from which it is straightforward to determine the complex angular velocity and spatial structure of each resonance.

Section 2 of the paper is concerned with the model equations and the method used for their solution in a region including the English Channel and Irish Sea. In Sect. 3 the model is tested by comparing its solution for the diurnal and semi-diurnal tides in the model region with observations.

Section 4 is concerned with the response of the model at real angular velocities, and this is extended in Sect. 5 to the complex angular velocity plane. Section 6 investigates the detailed structure of some of the key resonances, and shows that they form groups with very different properties. The insights are then used in Sect. 7 to show how the response at real frequencies depends on both the individual properties and the interactions of the different resonances.

\section{The numerical model}

A numerical model is used to solve Laplace's tidal equations. In vector notation and with a linear friction term these are

$$
\begin{aligned}
\partial \boldsymbol{u} / \partial t+\boldsymbol{f} \times \boldsymbol{u}+(\kappa / H) \boldsymbol{u}+g \nabla \zeta & =g \nabla \zeta_{\mathrm{e}}, \\
\partial \zeta / \partial t+\nabla \cdot(H \boldsymbol{u}) & =0 .
\end{aligned}
$$

$\boldsymbol{u}$ is the depth-averaged horizontal velocity, $t$ is time, $\zeta$ sea level, $\zeta_{\mathrm{e}}$ the height of the equilibrium tide (corrected for Earth tides), $\kappa$ the bottom friction coefficient, $g$ the acceleration due to gravity, $H$ the depth and " $\times$ " indicates a vector cross product. The model equations are solved for a latitudelongitude grid on a sphere. The Coriolis vector $\boldsymbol{f}$ is defined by

$\boldsymbol{f}=2 \Omega \cos (\theta) \boldsymbol{n}_{z}$,

where $\Omega$ is Earth's rotation rate, $\theta$ the co-latitude, and $\boldsymbol{n}_{z}$ the unit vertical vector. The equations are obtained by integrating the full equations of motion in the vertical and neglecting the vertical acceleration, non-linear and self-attraction terms.

The model assumes that the coastlines are fixed (i.e. that they do not change with the state of the tide) and that the normal velocity at a coastline is zero.

$\boldsymbol{u} \cdot \boldsymbol{n}=0$

The model can also have open boundaries at which Dirichlet, i.e. the tidal height $\zeta_{b}$, or more complex boundary conditions can be specified. The present study uses Dirichlet conditions. The possible effects of a Flather (1976) type radiational condition is covered in the discussion section.

Equation 1 contains a contribution from the equilibrium tide. In the case of the region studied later, the energy input from the boundary is much larger than that from the equilibrium tide. For the present study the equilibrium tide has therefore been set to zero.

Equations (1) and (3) are linear, so the general solution for a given forcing can be written as a linear combination of solutions of the form

$\left(\begin{array}{l}\boldsymbol{u}(t) \\ \zeta(t)\end{array}\right)=\Re\left[\left(\begin{array}{l}\boldsymbol{u} \\ \zeta\end{array}\right) \exp (-i \omega t)\right]$,

where $\omega$ is the angular velocity and $\Re$ represents the real part of the complex expression.

Substituting Eq. (4) in Eq. (1) and separating the vector $\boldsymbol{u}$ into its components $u$ and $v$ in the $x$ (east) and $y$ (north) directions:

$$
\begin{aligned}
(-i \omega+\kappa / H) u-f v+g \partial \zeta / \partial x & =0, \\
(-i \omega+\kappa / H) v+f u+g \partial \zeta / \partial y & =g, \\
-i \omega \zeta+\partial(H u) / \partial x+\partial(H v) / \partial y & =0 .
\end{aligned}
$$

These time-independent equations are the ones solved by the model. They allow for solutions to be obtained for both real and complex values of the angular velocity $\omega$.

\subsection{The finite-difference equations}

The equations are solved by first transforming them into their finite-difference equivalents, using an Arakawa $\mathrm{C}$ grid to specify the horizontal position of the variables $\zeta, u$ and $v$ (see Fig. 1). Such an approach is useful as long as the grid is fine enough that the values vary sufficiently smoothly between each grid point so that they are a good approximation both to the value at that point and to the average value within the surrounding grid cell.

Other arrangements of variables are possible, but the Arakawa $\mathrm{C}$ grid has the advantage that it offsets them in such a way as to most accurately represent the equations for long gravity waves.

Let $\zeta_{i, j}, u_{i, j}$ and $v_{i, j}$ represent one group of three such quantities where the indices $i$ and $j$ indicate their position on the grid. Substituting in Eq. (5),

$$
\begin{aligned}
(-i \omega+\kappa / H) u_{i, j}-(f v)_{u ; i, j}+g(\partial \zeta / \partial x)_{u ; i, j} & =0, \\
(-i \omega+\kappa / H) v_{i, j}+(f u)_{v ; i, j}+g(\partial \zeta / \partial y)_{v ; i, j} & =0, \\
-i \omega \zeta_{i, j}+(\partial(H u) / \partial x)_{\zeta ; i, j}+(\partial(H v) / \partial y)_{\zeta ; i, j} & =0,
\end{aligned}
$$

where the subscripts " $u ; i, j$ ", indicate the value of the quantity at the point $u_{i, j}$ and similarly for " $v ; i, j$ " and " $\zeta ; i, j$ ". 
The Arakawa $\mathrm{C}$ grid allows for the horizontal gradients to be represented in terms of symmetrically placed neighbouring values:

$$
\begin{aligned}
(\partial \zeta / \partial x)_{u ; i, j} & =\left(\zeta_{i+1, j}-\zeta_{i, j}\right) / d x_{i, j}^{*}, \\
(\partial \zeta / \partial y)_{v ; i, j} & =\left(\zeta_{i, j+1}-\zeta_{i, j}\right) / d y_{i, j}^{*}, \\
(\partial(H u) / \partial x)_{\zeta ; i, j} & =\left(H_{u ; i, j} u_{i, j}-H_{u ; i-1, j} u_{i-1, j}\right) / d x_{i, j}, \\
(\partial(H v) / \partial x)_{\zeta ; i, j} & =\left(H_{v ; i, j} v_{i, j}-H_{v ; i, j-1} v_{i, j-1}\right) / d y_{i, j},
\end{aligned}
$$

where $H_{u ; i, j}$ is the depth of the $u_{i, j}$ grid cell, and the grid spacings $d x_{i, j}, d y_{i, j}, d x_{i, j}^{*}$ and $d y_{i, j}^{*}$ are defined in Fig. 1. These approximations are correct to $O\left(d x^{2}\right)$ and $O\left(d y^{2}\right)$, so the errors reduce rapidly as the grid size is reduced. It is also straightforward to show that they conserve the total sum of kinetic and potential energy.

For the Coriolis term, conservation of energy requires special consideration. This is discussed briefly in Appendix A.

\subsection{Boundary conditions}

The coastline of the finite-difference model is assumed to follow the boundaries of the grid cells representing the variables $\zeta$. The arrangement of variables on the Arakawa $\mathrm{C}$ grid then means that each such section of coastline should pass through either one of the $u_{i, j}$ or $v_{i, j}$ grid points. As there is no flow through coastlines (Eq. 3 ) the velocity at such grid points is zero.

The present model also allows for the tidal heights $\zeta$ to be specified at an open boundary. Neighbouring velocity points which lie within the model region are handled as normal. The velocity point between two open boundary $\zeta$ points is set to the value one row in. This prevents jumps in the Coriolis term adjacent to the open boundary.

A small program was used to convert the GEBCO coastline and the GEBCO depth data (IOC et al., 2003) into the datasets defining the model coastline and depth field. The GEBCO depth data is at a higher resolution $\left(1 / 60^{\circ}\right)$ than that used for the model grid, so depths were calculated such that the volume of each model grid cell was the same as the corresponding GEBCO region.

\subsection{The band matrix equation}

Each finite-difference equation that results from substituting Eqs. (7), (A1) and the boundary conditions into Eq. (6) connects variable values at one point on the grid $(i, j)$ to values at neighbouring grid points $(i \pm 1, j \pm 1)$. If the variables $u_{i, j}$, $v_{i, j}$ and $\zeta_{i, j}$ are ordered as a single vector $\boldsymbol{x}$, then this can be expressed as a matrix equation:

$\mathbf{M x}=\boldsymbol{b}$,

where $\mathbf{M}$ is a sparse matrix containing the coefficients generated by the finite-difference equations and $\boldsymbol{b}$ is a vector, zero except for the variables representing the tidal height on the open boundary. If the numbering is systematic, $\mathbf{M}$ becomes

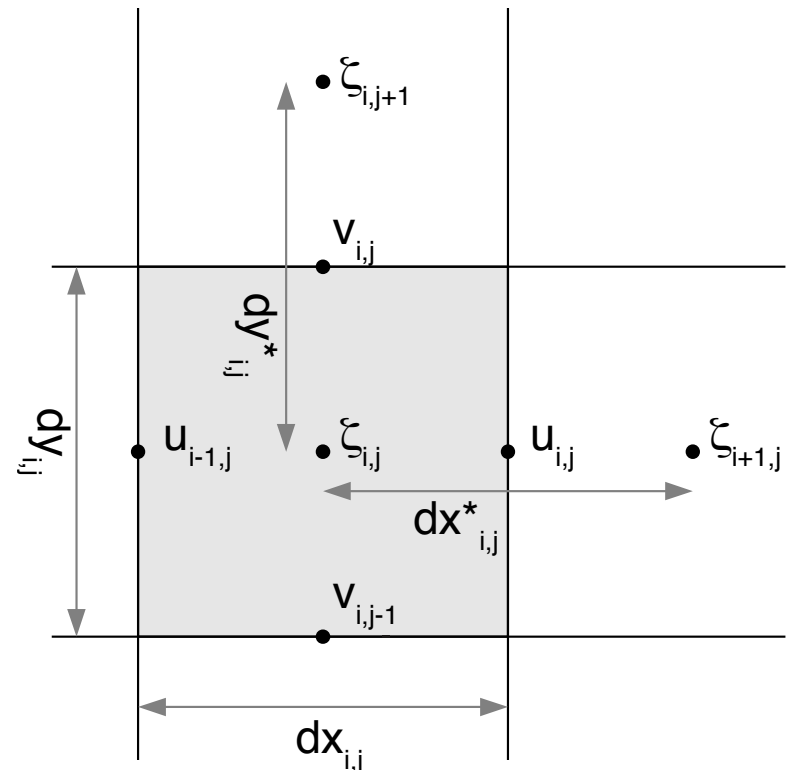

Fig. 1. The Arakawa $\mathrm{C}$ grid, showing the relative position of sea level grid points $(\zeta)$ and the easterly $(u)$ and northerly $(v)$ components of velocity. The shaded area is the "grid cell" surrounding the point $\zeta_{i . j}$. The distances $d x_{i, j}$ etc. are those of Eq. (7).

a band matrix (i.e. one which is clustered around the diagonal), the width of the band depending only on the maximum separation in index between neighbouring variables.

In the model results discussed later, the vector $\boldsymbol{x}$ contains 49500 variables. If this is represented by $n$, then a full solution of a similar densely packed matrix equation would need of the order of $n^{3}$ operations ( $1.2 \times 10^{14}$ operations), which becomes computationally expensive. However, band matrix equations can be solved in order $n m^{2}$ operations, where $m$ is the width of the band. Minimizing $m$ thus greatly minimizes the cost of the calculation.

In the model discussed here there are fewer grid cells in the north-south direction than in the east-west direction. The width of the band was therefore minimized by ordering the elements of $x_{k}$ such that $\zeta_{i, j}, u_{i, j}$ and $v_{i, j}$ are adjacent, and followed either by $\zeta_{i, j+1}, u_{i, j+1}$ and $v_{i, j+1}$ or, if the top of column $i$ has been reached, by the corresponding terms at the bottom of the next column.

The resulting band matrix has a width of approximately 850 elements. It was stored in the compressed band matrix form used by the BLAS and LAPACK libraries, and was solved using the LAPACK band matrix subroutine "zgbsv". This took about $25 \mathrm{~s}$ using optimized versions of the routines developed by the manufacturers for both Intel and AMD processors. 

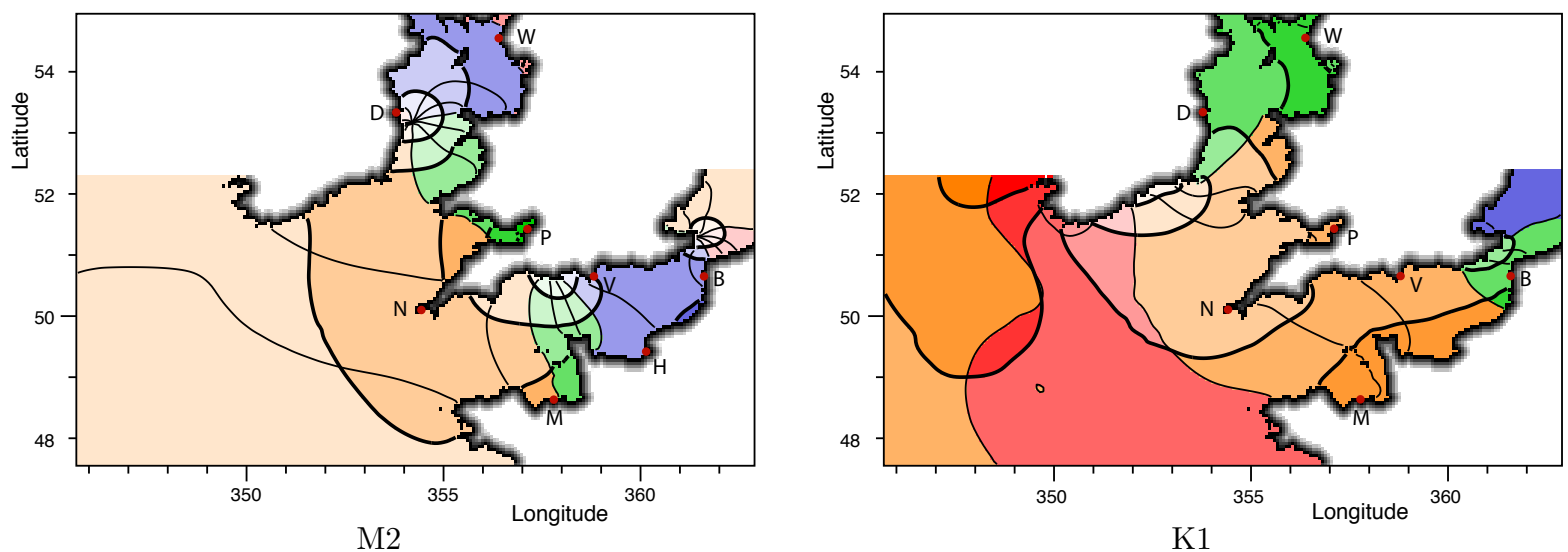

Fig. 2. The model $\mathrm{M} 2$ and $\mathrm{K} 1$ tides. Thick lines are contours of amplitude; $\mathrm{M} 2$ at $0.5 \mathrm{~m}, 1.5 \mathrm{~m}$ and intervals of $1.5 \mathrm{~m}$; $\mathrm{K} 1 \mathrm{at} 1 \mathrm{~cm}, 2.5 \mathrm{~cm}$ and intervals of $2.5 \mathrm{~cm}$. In the southwest the M2 amplitude is $0.87 \mathrm{~m}$ and $\mathrm{K} 1$ is $6.4 \mathrm{~cm}$. Thin lines are contours of phase at intervals of $30^{\circ}$.

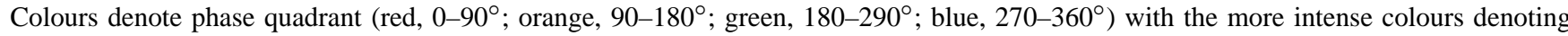
higher amplitudes. The tide gauge stations are D, Dublin; W, Workington; P, Portishead; N, Newlyn; V, Ventnor; B, Boulogne; H, Le Havre; M, St. Malo.

\section{Comparison with observations}

The model was checked by using it to reproduce the M2 and K1 tides and comparing the results against tide gauge observations. The only free-model parameters are the position and resolution of the grid, the tidal heights on the open boundary, the minimum depth in any grid cell and the coefficient of linear bottom friction. For the M2 tide a bottom friction of $0.2 \mathrm{~cm} \mathrm{~s}^{-1}$ was found to be best. This is double the value used by Webb (1981), and may result from the higher tides and stronger currents in the English Channel and Irish Sea. The minimum depth was set to $2.5 \mathrm{~m}$, the same as used in Webb (1981).

Initially tests were carried out with a model resolution of $0.1^{\circ}$ in the north-south direction and $0.155^{\circ}$ east-west. However this was found to produce a truncated Bristol Channel, and as the semi-diurnal tides of the Bristol Channel are of great interest, a resolution of $0.05^{\circ} \times 0.0755^{\circ}$ was used for the results presented here.

The open boundary to the west (see Fig. 2) was chosen to lie well beyond the shelf edge because of its possible importance in generating resonances. The tidal amplitude and phases on the boundary were taken from the map of Cartwright et al. (1980a), which is based on coastal and deep sea tide gauges.

Initially, closed boundaries were placed in both the Dover Strait and the North Channel of the Irish Sea. This was done partly to reduce the computational cost but also on the basis that only a small fraction of the tidal energy was passing through the straits. If this is so, then the barriers should have little effect on the tides and resonances of the English Channel and Irish Sea.

However this arrangement resulted in a maximum of the M2 tide in the Dover Strait. As this might be unrealistic, a number of tests were made extending the model into the North Sea. It was found that as long as the closed boundary was some distance beyond the Dover Strait, the amplitude in the Dover Strait was significantly reduced. The tidal amplitude also dropped off rapidly once through the Dover Strait, confirming that with these more realistic boundaries only a small amount of energy passes through the Dover Strait.

The closed boundary in the northwest of the Irish Sea appeared to have little effect on the tides of the region, and was therefore not modified.

\subsection{The M2 tide}

The M2 tide calculated by the model is shown in Fig. 2, and the amplitudes and phases at key points are compared with tide gauge measurements in Table 1. Figure 2 shows that qualitatively the model is in good agreement with the observed M2 tide in the region. It reproduces the high tides of the Bristol Channel and the Gulf of St. Malo and also the virtual amphidromes near Dublin and Southampton. Local maxima are also found in the northeast Irish Sea and at the eastern end of the Channel near Boulogne.

The individual values given in Table 1 show reasonable agreement, but the model could do better. The best agreements are found along the English Channel at Boulogne, Le Havre and St. Malo. Workington in the Irish Sea is also acceptable, but at Portishead in the Bristol Channel, the amplitude is much too large. The phase error there is also much larger than that seen in Gulf of St. Malo, where the amplitude is also large.

In the study, discussed earlier, where the grid spacing was double the present value, the amplitude of the M2 tide at the head of the estuary was far too small. It appears therefore that 
Table 1. Amplitude and phase of M2 tide calculated by the model compared with observations at representative tide gauges.

\begin{tabular}{lrrrr}
\hline Tide Gauge & \multicolumn{2}{c}{ Model Tide } & \multicolumn{2}{c}{ Observed Tide } \\
& $\begin{array}{r}\text { Amplitude } \\
(\mathrm{m})\end{array}$ & $\begin{array}{r}\text { Phase } \\
(\mathrm{deg})\end{array}$ & $\begin{array}{r}\text { Amplitude } \\
(\mathrm{m})\end{array}$ & $\begin{array}{r}\text { Phase } \\
(\mathrm{deg})\end{array}$ \\
\hline Dublin & 0.46 & 331 & 1.33 & 325 \\
Workington & 2.80 & 358 & 2.66 & 333 \\
Portishead & 8.42 & 221 & 4.20 & 201 \\
Newlyn & 2.17 & 149 & 1.72 & 134 \\
Ventnor & 1.64 & 310 & 0.97 & 311 \\
Boulogne & 2.76 & 343 & 2.93 & 327 \\
Le Havre & 2.87 & 291 & 2.62 & 296 \\
St. Malo & 3.35 & 198 & 3.69 & 178 \\
\hline
\end{tabular}

the tides in the Bristol Channel are very sensitive to changes in the physical system.

\subsection{The K1 tide}

When the same procedure was used for the $\mathrm{K} 1$ tide, the results for the English Channel and Irish Sea were reasonable, but the model generated unusual features near the continental shelf slope. It also generated unrealistically high tides to the west of Ireland. On investigation the problem appeared to be continental shelf waves, excited by the K1 tide, which could not pass through the northern section of the open boundary. The study by Cartwright et al. (1980b) indicated that continental shelf waves have a significant impact on the K1 tide to the west of the British Isles.

The problem was overcome by extending the model region to include the whole of the British Isles and the North Sea, again using open boundary forcing from Cartwright et al. (1980a). This time the tidal heights to the west of Ireland were reasonable.

A revised solution for the standard model region was then calculated using values on the open boundary taken from the larger model. The result is shown in Fig. 2 with individual values compared with observations in Table 2 . With the exception of Boulogne, which may be affected by the tides of the North Sea, the phases are reasonable but the amplitudes are low, possibly a further effect of processes near the open boundary.

In reviewing these results it was decided that, although the comparisons show that the model M2 and K1 tides are not perfect, they were sufficiently realistic for the present preliminary study. As a result the results presented in the following sections use the same model with changes only in the open boundary conditions and in the frequency of the forcing.
Table 2. Amplitude and phase of K1 tide calculated by the model compared with observations at representative tide gauges.

\begin{tabular}{lrrrr}
\hline Tide Gauge & \multicolumn{2}{c}{ Model Tide } & \multicolumn{2}{c}{ Observed Tide } \\
\hline & $\begin{array}{r}\text { Amplitude } \\
(\mathrm{m})\end{array}$ & $\begin{array}{r}\text { Phase } \\
(\mathrm{deg})\end{array}$ & $\begin{array}{r}\text { Amplitude } \\
(\mathrm{m})\end{array}$ & $\begin{array}{r}\text { Phase } \\
(\mathrm{deg})\end{array}$ \\
\hline Dublin & 0.06 & 210 & 0.10 & 190 \\
Workington & 0.08 & 207 & 0.12 & 194 \\
Portishead & 0.05 & 128 & 0.07 & 142 \\
Newlyn & 0.04 & 108 & 0.06 & 110 \\
Ventnor & 0.06 & 163 & 0.09 & 103 \\
Boulogne & 0.07 & 192 & 0.05 & 123 \\
Le Havre & 0.09 & 159 & 0.09 & 119 \\
St. Malo & 0.08 & 114 & 0.10 & 097 \\
\hline
\end{tabular}

\section{The response function}

For studying the model response functions, the forcing at the open boundary was changed so that the tide there had unit amplitude and zero phase. The model was then run at intervals of $0.1 \mathrm{rad} \mathrm{day}^{-1}$ (radians per day) between zero and $30 \mathrm{rad} /$ day $(\sim 4.8$ cycles per day).

The response function $R(\omega)$ is defined as

$R(\omega)=\zeta(\omega) / \zeta_{\mathrm{b}}$,

where $\zeta(\omega)$ is the (complex) tidal height at station $x$ and $\zeta_{\mathrm{b}}$ is the tidal height on the open boundary.

Figure 3 shows the resulting response function amplitudes at four representative stations. Portishead, in the Bristol Channel, and St. Malo, in the Bay of St. Malo, are included because the tides in these regions are high. Workington and Le Havre are included as being more typical of the rest of the Irish Sea and English Channel.

All the stations have amplitudes near 1 at low angular velocities. At very low angular velocities this is inevitable because the system always has time to reach equilibrium with the forcing. As angular velocity increases Rossby waves resonances can, in principle, be excited. However, their effect on sea level is small, and in shallow seas their decay rate is likely to be faster than both their angular velocity and their separation. As a result a smooth response at low angular velocities is not unexpected.

As angular velocity increases, the figure shows marked differences in the response at the different stations. At Portishead in the Bristol Channel, the amplitude of the response function initially drops, but it then rises rapidly to a peak between 13 and $14 \mathrm{rad} \mathrm{day}^{-1}$. After this it continues rising, past an initial low peak and then two much higher peaks between 20 and $30 \mathrm{rad} / \mathrm{day}$.

In contrast, the response of Workington, in the Irish Sea, remains low throughout the range studied. It shows a small low peak between 5 and $6 \mathrm{rad} /$ day and two other similar peaks near 13 and $21 \mathrm{rad} /$ day before falling to near zero. 


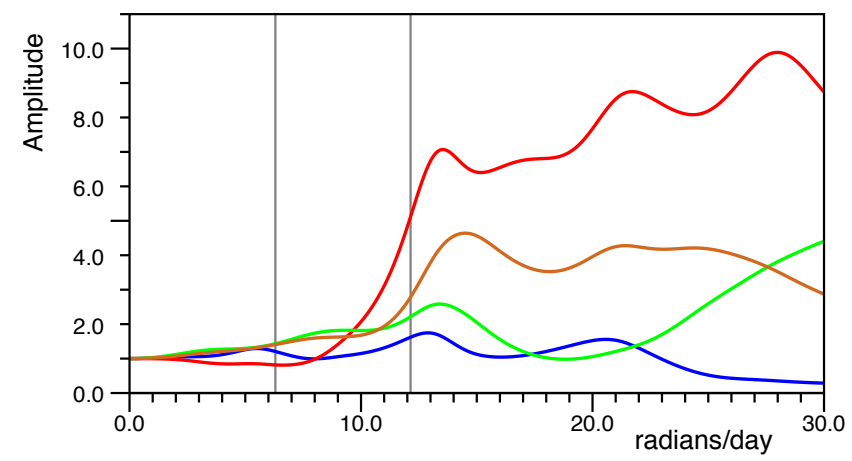

Fig. 3. The response function amplitude as a function of angular velocity for Portishead (red), St. Malo (brown), Workington (blue) and Le Havre (green). Vertical lines indicate the angular velocities of the $\mathrm{K} 1$ and $\mathrm{M} 2$ tides.

The two English Channel stations show an initial gradual rise in amplitude, with possible low, broad resonances around 4 and 8 rad day $^{-1}$. St. Malo then increases to a peak around $14 \mathrm{rad} \mathrm{day}^{-1}$ and continues at only slightly lower amplitudes to $25 \mathrm{rad} /$ day after which it starts decaying. At Le Havre there is a lower peak, around $13 \mathrm{rad} /$ day, followed by a drop in amplitude and then a steady climb to beyond $30 \mathrm{rad} /$ day at which point the response amplitude is larger than that at St. Malo.

Note that although the different stations often have maximum amplitudes at nearby values of angular velocity, the differences are rarely small enough to be sure that only a single resonance is involved. Thus all four stations show peaks in the regions of 13 and $14 \mathrm{rad} /$ day, but the Workington and St. Malo maxima have a significant offset.

Webb (2012) investigated other ways of representing the tidal response function and showed that a polar plot, in which the real and imaginary components are plotted against each other, was often the most informative. This is because each resonance contributes a single anti-clockwise circular loop. Thus loops in the polar plot can result from the contribution of a single strong resonance. However less regular loops can also be generated by groups of nearby resonances.

Figure 4 shows the polar plots for the four locations of Fig. 3. Each locus starts at the equilibrium response point $(1,0)$ and moves initially in an anti-clockwise direction. The rate of change of the angle relative to the origin is a measure of the time taken for a signal to propagate from the open boundary to each station (Webb, 1973).

At Portishead there is a small loop, near the diurnal tidal band, which is followed by a series of very large loops. The amplitude plot shows what appears to be a series of overlapping resonances, and the polar plot supports this view. It is also noticeable that at angular velocities above the semidiurnal tidal band, the curve is roughly circular. This is the form one might expect in a system in which the main feature
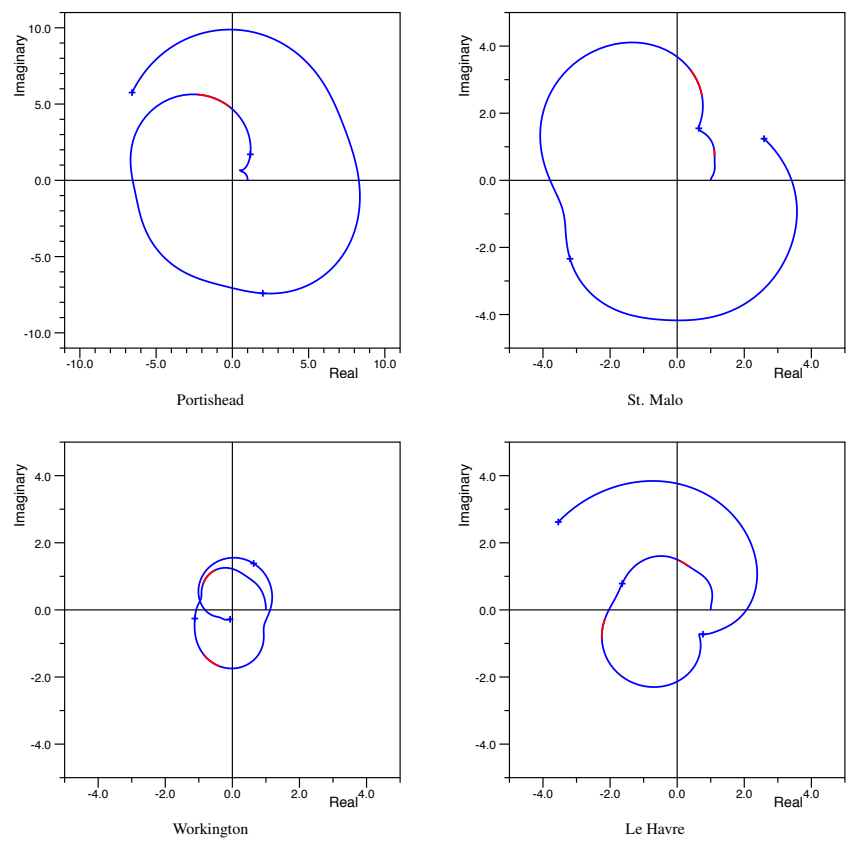

Fig. 4. Polar plots showing the real and imaginary components of the response function at Portishead, St. Malo, Workington and Le Havre in the range 0 to $30 \mathrm{rad} /$ day. The function has the value $(1,0)$ at the origin. The crosses mark multiples of $10 \mathrm{rad} \mathrm{day}^{-1}$. The red sections indicate the position of the diurnal and semi-diurnal tidal bands.

is a pure delay, but it is unexpected for what is understood to be a resonant Bristol Channel.

At St. Malo amplitudes are lower and the initial loop extends further to $10 \mathrm{rad} / \mathrm{day}$. There is then a loop extending past the semi-diurnal band and two further loops in the region extending to $30 \mathrm{rad} \mathrm{day}^{-1}$. Again above the semidiurnal band there is a tendency to follow a semi-circular path around the origin, but this is not so marked as at Portishead.

At Workington the response function appears to be made up primarily of three resonant contributions. The first dominates between 0 and $10 \mathrm{rad} \mathrm{day}^{-1}$. There is then a second loop with opposite phase and a final one which culminates in a very low amplitude near $30 \mathrm{rad} \mathrm{day}^{-1}$. The response at Le Havre is also dominated by three loop structures, but the first is at a slightly higher angular velocity relative to the diurnal band. The second loop may be similar but the third produces a final increase in amplitude near $30 \mathrm{rad} \mathrm{day}^{-1}$.

Overall these results indicate that there may be between 8 or 10 resonances that affect the tides between 0 and $30 \mathrm{rad} /$ day. Time-stepping models, which can only be forced with real values of angular velocity, may give some information on their properties. However, the results show that the resonances overlap so methods such as analytic continuation are needed to separate their individual properties. Analytic continuation using power series should work for 


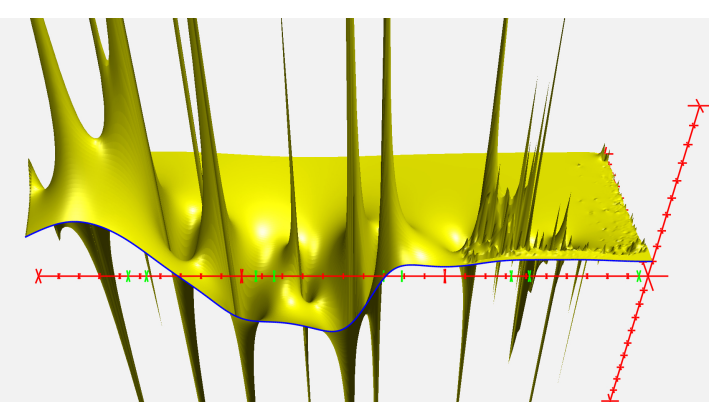

Real

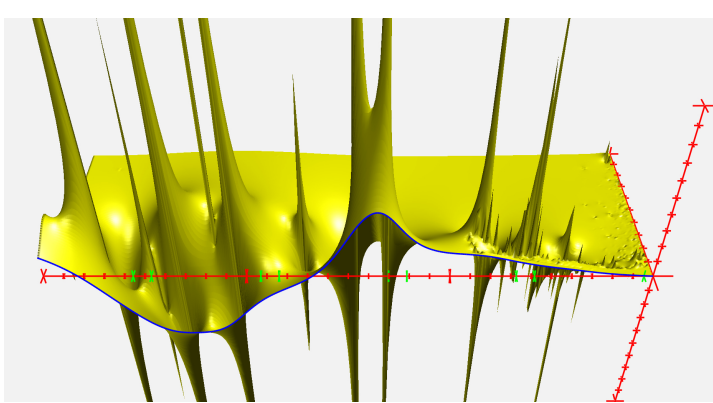

Imaginary

Fig. 5. The real and imaginary components of the response function plotted as a function of complex angular velocity at St. Malo. The origin $(0,0)$ is on the right with the positive real axis (in red) running from right to left and the negative imaginary axis running into the figure. Function values on the real axis are plotted in blue. The axes have red crosses every $1 \mathrm{rad} / \mathrm{day}$. On the real axis green crosses indicate the limits of the tidal bands near 0 (long period), 1 (diurnal), 2 (semi-diurnal), 3 and 4 cycles per day.

isolated resonances near the real axis, but in more complex situations, as may exist here, other methods will be required. Any method using analytic continuation will also be affected by numerical rounding errors within the models.

\section{The complex $\omega$ plane}

The great advantage of the present model is that it allows for a direct investigation of the complex plane. Figures 5 and 6 show the real and imaginary parts of the response functions for St. Malo and Portishead for the rectangular region bounded by the origin and the points $(0-10 i),(30-10 i)$ and $30 \mathrm{rad} /$ day. Within the region the response function was calculated at real and imaginary intervals of $0.1 \mathrm{rad} /$ day.

The real axis runs from right to left along the front of the figure. As discussed by Webb (1973) and Webb (2012), response functions are analytic functions with a pole at the angular velocity of each resonance of the system. In the neighbourhood of each resonance the response function has the form

$R(\omega)=R_{i} /\left(\omega-\omega_{i}\right)+B(\omega)$,

where $\omega_{i}$ is the angular velocity of the resonance and $R_{i}$ is its residue. $B(\omega)$ is the smooth background representing the contributions from distant poles.

The form of this equation means that the magnitude of $R(\omega)$ tends to infinity as $\omega$ approaches $\omega_{i}$, the rate at which this happens depending on the residue $R_{i}$. If the magnitude is $R_{i}$ is small, the very large values around a resonance may not be represented in the grid of calculated values used to construct this figure. In this case the resonance shows up as a small peak.

The resolution of the main figures is insufficient to show all the detail near the origin, and so Fig. 7 shows the Portishead response function (real component) near the origin at a resolution of $0.02 \mathrm{rad} \mathrm{day}^{-1}$.
All the figures show two types of resonances. The first is a series of large peaks which start around $6 \mathrm{rad} /$ day and continue to higher angular velocities. The second is a dense set of much smaller resonances found close to the real axis below $10 \mathrm{rad} /$ day and along the negative imaginary axis. Fig. 7 shows that many of the second set of resonances form densely packed lines, and there is often a step change in the response function between one side of the line and the other.

In the region studied, the Coriolis term, $f$, has a value near $1.210^{-4} \mathrm{~s}^{-1}\left(\sim 10 \mathrm{rad} \mathrm{day}^{-1}\right)$. This is near the upper limit of the band where the second set of resonances is found. In order to understand how it affected the solution, the calculations were repeated, first with the Coriolis term halved and then with it set to zero.

The changes in the Coriolis term had little effect on the resonances at above $10 \mathrm{rad} /$ day, but at low angular velocities all but three of the resonances moved to the imaginary axis. This implies that the first set of resonances and three of the second set are primarily gravity waves. The remainder must be either Rossby waves, which depend on the gradient of the Coriolis term $f$, or topographic waves, such as continental shelf waves, which depend on the gradient of $f / h$, where $h$ is depth.

The low angular velocity resonances that remain when $f$ is zero are resonances $\mathrm{A}$ and $\mathrm{C}$ of Table 3 plus a resonance with a complex angular velocity of approximately $(6-i 1) \mathrm{rad} / \mathrm{day}$. This is near the point in Fig. 7 where the largest line of Rossby/topographic resonances breaks up into a short series of isolated peaks. It is thus likely that the line of larger peaks around $(6-i 1) \mathrm{rad} /$ day results from coupling between this gravity wave resonance and nearby Rossby/topographic waves.

\subsection{Resonance angular velocities}

The angular velocity of the resonances can be found by fitting Eq. (10) to the response function in the neighbourhood of each resonance. Assuming the background term is 

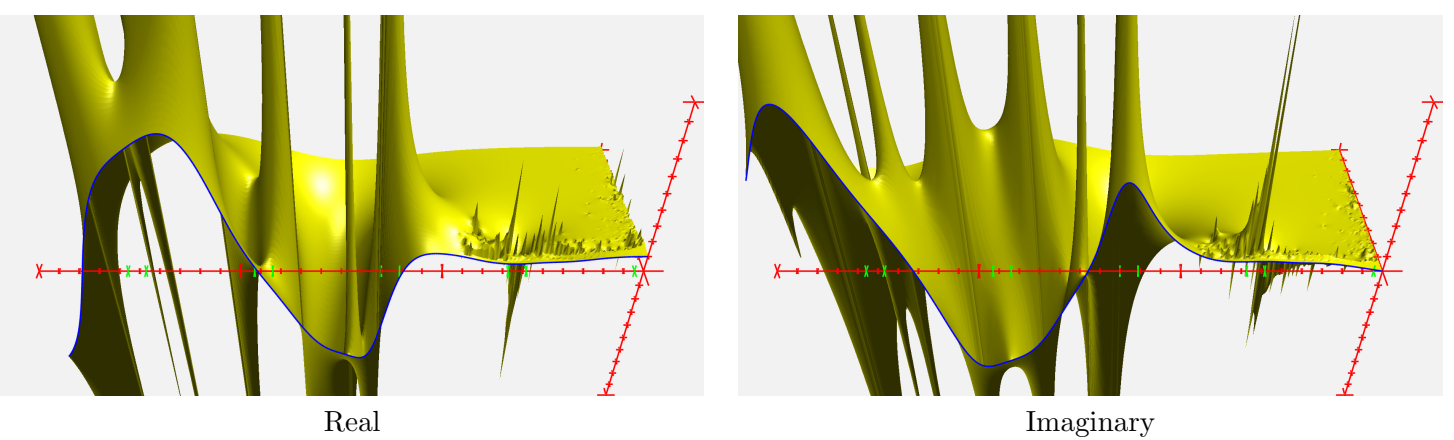

Fig. 6. The real and imaginary components of the response function at Portishead. Axes as in Fig. 5

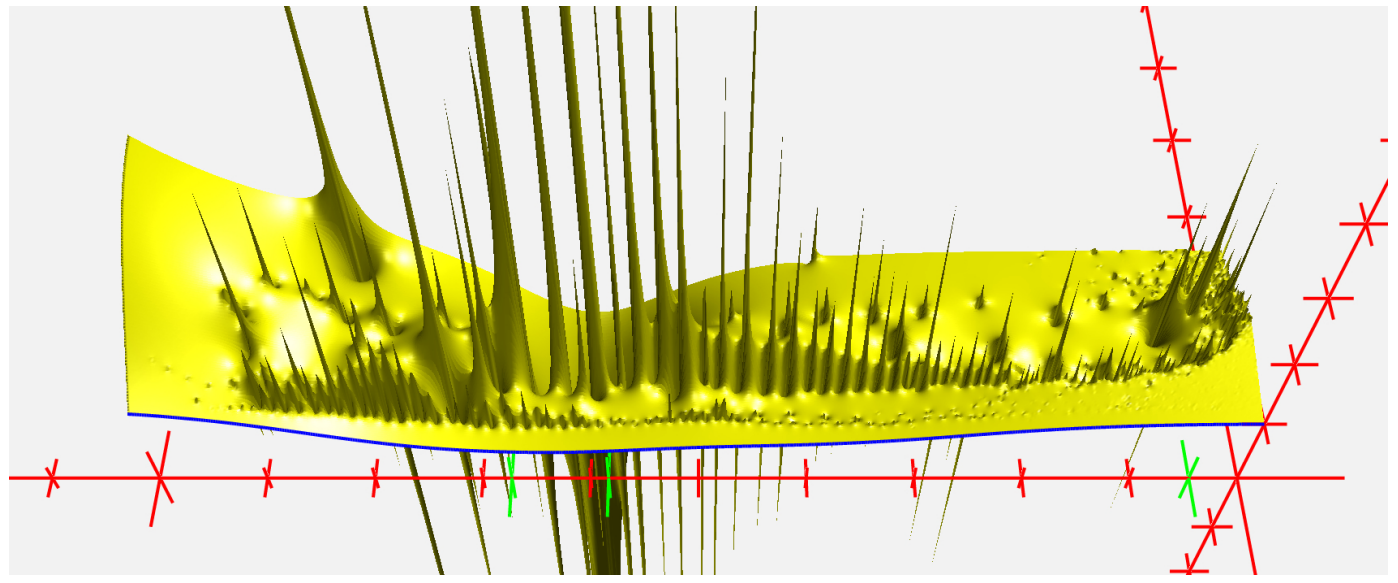

Fig. 7. Close-up near the origin (region $(0,0)$ to $(5,-2)$ ) of the real component of the response function at Portishead. Axes as in Fig. 5

approximately linear

$R(\omega)=R_{j} /\left(\omega-\omega_{j}\right)+A+B \omega$.

As discussed in Webb (2012), this can be converted into a linear equation. If the value of $R(\omega)$ is available for four values of $\omega$, the resulting matrix equation can be solved for the four unknowns including the resonance frequency $\omega_{j}$.

Table 3 contains results for the main gravity wave resonances and the major members of the second set of resonances near $(6-i 1) \mathrm{rad} /$ day. Initial estimates of resonance positions were made using the datasets used to construct the Portishead response functions (Figs. 6 and 7). The method was then iterated, first calculating the full solution for the four values of $\omega$ separated by \pm 0.02 and $\pm i 0.02 \mathrm{rad} /$ day from the previous best estimate. Equation (11) was then solved for the model grid point at which the response function amplitude was largest, and thus might be expected to be largest relative to the background, in the first of these solutions.

At angular velocities near $10 \mathrm{rad} /$ day the gravity resonances are well separated and have imaginary components of angular velocity around -1.6 to $-2 \mathrm{rad} /$ day. The latter implies that their decay times are around 12 to 15 hours. At higher real angular velocities the resonances tend to be closer together and also have larger imaginary components, the decay time dropping to $8 \mathrm{~h}$ or less.

Webb (2011) showed that when the resonances are well separated and only have small imaginary components, the real-world response is dominated by a few nearby resonances. Where the resonances are close relative to their distance from the real axis, cooperative effects become important.

Interpreting the 3-D response function figures in this way, the semi-diurnal tidal band, near $12 \mathrm{rad} /$ day, is dominated by the resonances D and E. In the Bristol Channel, at Portishead, $\mathrm{D}$ is much the stronger of the two but at St. Malo they appear to have roughly equal effect on the response function.

Previous studies of the Bristol Channel have concluded that the large semi-diurnal tides are due to a resonance, but this appears to be the first time that a model suggests that two nearby resonances are involved and that the high tides of the Bristol Channel and the Gulf of St. Malo involve the same resonances.

At lower values of angular velocity, although the resonances are closer to the real axis, the response function along the axis is very smooth. Individually the resonances are having little effect, but the step change associated by the lines of 
Table 3. Real and imaginary components of angular velocity (in radians per day) for the gravity wave resonances and the major resonances found near the location of resonance $B$, calculated using the method described in the text. B is a single gravity wave resonance when the Coriolis term is zero but is mixed with nearby continental shelf wave modes when the Coriolis term has the correct value.

\begin{tabular}{rrrrrr}
\hline & \multicolumn{2}{c}{ Angular velocity } & \multicolumn{2}{c}{ Angular velocity } \\
\hline & Real & Imag. & & Real & Imag. \\
\hline $\mathrm{A}$ & 3.9057 & -2.0017 & $\mathrm{D}$ & 13.1387 & -1.6323 \\
$\mathrm{Ba}$ & 5.3612 & -0.7935 & $\mathrm{E}$ & 14.6609 & -2.0767 \\
$\mathrm{Bb}$ & 5.5931 & -0.8501 & $\mathrm{~F}$ & 17.4442 & -3.1087 \\
$\mathrm{Bc}$ & 5.7544 & -0.8720 & $\mathrm{G}$ & 21.0201 & -2.2989 \\
$\mathrm{Bd}$ & 5.9688 & -0.9047 & $\mathrm{H}$ & 22.0992 & -3.3991 \\
$\mathrm{Be}$ & 6.2037 & -0.8680 & $\mathrm{I}$ & 24.2708 & -2.9581 \\
$\mathrm{Bf}$ & 6.5763 & -0.8760 & $\mathrm{~J}$ & 25.5625 & -3.1394 \\
$\mathrm{C}$ & 8.2549 & -1.8567 & $\mathrm{~K}$ & 27.8690 & -2.9693 \\
\hline
\end{tabular}

resonances is large, and this is likely to have some effect on the response along the real axis.

\section{Spatial behaviour}

Following Webb (2012), the spatial structure can be calculated in a similar manner. Solutions to the model equations were obtained for each of the four angular velocities $\omega_{j} \pm \delta \omega$ and $\omega_{j} \pm \imath \delta \omega$, where $\delta \omega$ equals $0.02 \mathrm{rad} / \mathrm{day}$. The four solutions were then fitted, at all points in the model grid, to a function similar to that of Eq. (11) but where the resonance angular velocity $\omega_{j}$ is fixed and the background is a quadratic function. The spatial variation of $R_{j}$ is then the $j$-th resonance eigenfunction of the model.

The resulting eigenfunction for the two resonances near the semi-diurnal tidal band are shown in Fig. 8. The functions are normalized so that their maximum amplitude equals 1 . In each case the amplitude on the open boundary is zero and it remains low until the continental shelf is reached.

Resonance D has the form of quarter-wave resonance of the Bristol Channel. In this mode amplitudes greater than $80 \%$ of the maximum are confined to a small region of the Bristol Channel itself. There is a second maximum in the Gulf of St. Malo, the phase at St. Malo leading that at Portishead by $63^{\circ}$ but with only 0.24 of the amplitude.

Lower amplitude standing waves are found in the English Channel and Irish Sea, and there are nodes on the lines between Anglesey and Dublin and between Cherbourg and Portland. The positions of the nodes are similar to those of the amphidromes of the actual semi-diurnal tides.

Resonance E shows similar standing waves in the English Channel and Irish Sea, but now the amplitudes in the Bristol Channel and the Gulf of St. Malo are similar. At St. Malo itself the wave height is 0.88 of that at Portishead and, instead of leading, now lags Portishead by $108^{\circ}$. This corresponds to a change of almost $180^{\circ}$ from mode $\mathrm{D}$.

Higher modes have a more complicated structure, and it is more difficult to relate the modes to specific physical features of the system. However, resonance F (Fig. 9) again has its highest amplitude at the head of the Bristol Channel, and this is also true for mode G. In the latter case there are also large amplitudes in Cardigan Bay and the northern Irish Sea indicating a possible complex interaction between the different bays within the region.

Two of the B resonances, which are close to the diurnal tidal band, are also shown in Fig. 9. These have been scaled so that the solution in the Irish Sea and English Channel regions are visible. A feature of all the low angular velocity modes investigated was a series of short-wavelength features along the continental slope and a region of very high amplitudes along the southern boundary of the model. These are seen in both figures. When the Coriolis term is set to zero, the mode $\mathrm{B}$ which remains with a similar angular velocity does not show either of these features. Instead it has the form of a simple quarter-wave resonance of the Irish Sea.

The position and wavelength of the oscillations on the continental slope indicate that they are almost certainly due to continental shelf waves. The behaviour at the southern end of the shelf is physically unrealistic and presumably arises because the boundary condition used is incompatible with the normal propagation of these waves.

\subsection{Continental shelf waves}

The theory of continental shelf waves usually assumes the shelf is infinitely long. Using this approximation, Huthnance (1975) and Brink (1991) showed that the waves form groups each with different dispersion curves. Each curve represents a set of waves with a complete range of longshore wavenumbers but all with a similar cross-shelf structure (i.e. the same number of zero crossings of the height field)

Experience in other areas of physics (Webb, 1976) shows that such dispersion curves produce cuts in a multi-valued analytic response function and that, in the equivalent finite systems, each cut becomes replaced by a line of resonances with similar properties.

The lines of closely spaced resonances seen in Fig. 7 fit this picture in that in most cases they also show a step change in the response function between the two sides of the line, similar to that due to a cut. Other resonances at low angular velocity are likely to be Rossby waves, but other topographic waves may also be present.

\section{The resonance contributions to the response function}

Previous studies (Webb, 2012, 2011) showed that at real values of the angular velocity it was possible to understand the structure of the response function in terms of the changing 


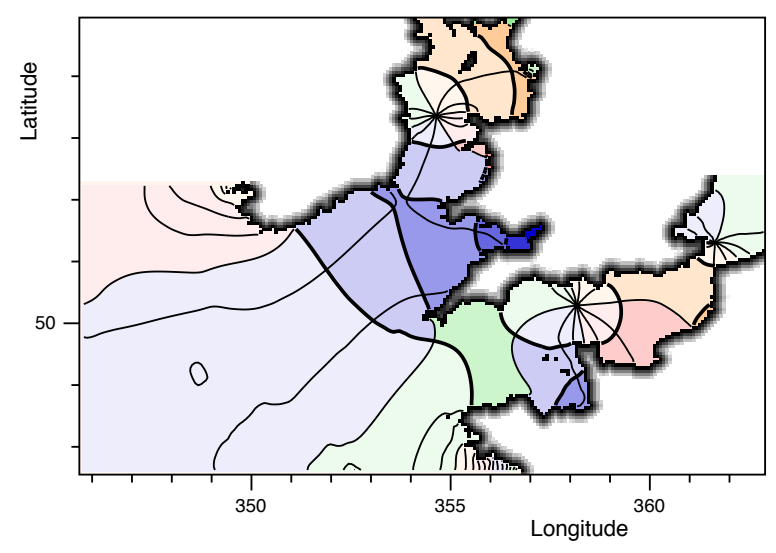

Resonance D

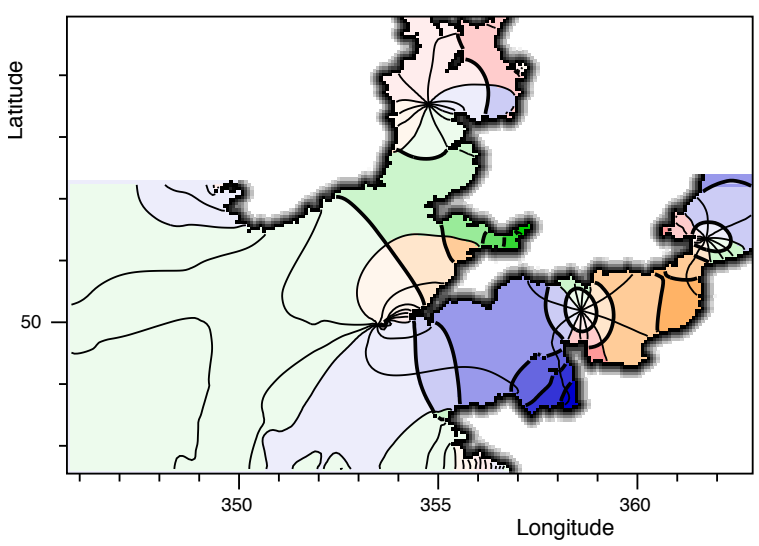

Resonance E

Fig. 8. Amplitude and phase of the resonances $\mathrm{D}$ and $\mathrm{E}$ (Table 3). The resonance is normalized such that its maximum amplitude is 1.0 . Colours denote phase (red, $0-90^{\circ}$; brown, $90-180^{\circ}$; green, $1800-270^{\circ}$; blue, $270-360^{\circ}$ ) with additional thin contours plotted at intervals of $30^{\circ}$. Colour intensity denotes amplitude. The lightest colours, seen near the shelf edge and beyond, denote amplitudes less than $1 \%$ of the maximum. The remaining bands separated by thick contours denote $1-20 \%, 20-40 \%, 40-60 \%, 60-80 \%$ and $80-100 \%$ of the maximum.

contributions from nearby resonances. Thus in the neighbourhood of each tidal band, the response function $R(x, \omega)$ at site $x$ is expanded in the form

$R(x, \omega)=\sum_{j} R_{j}(x) /\left(\omega-\omega_{j}\right)+S(x, \omega)+B(x, \omega)$,

where the sum $j$ is over the key nearby resonances. The residues $R_{j}(x)$ and the resonant angular velocities $\omega_{j}$ are the values calculated in the previous section. The symmetry term $S(x, \omega)$ is not essential but it is easy to calculate and represents the contribution from the conjugate set of poles ${ }^{1}$ with angular velocities $-\omega_{j}^{*}$ and residues $-R_{j}^{*}(x)$. The final term $B(x, \omega)$ represents the smooth background due to the remaining distant resonances. In (Webb, 2012) this was found to be generally small.

Figure 10 shows how the contributions from resonances $\mathrm{C}, \mathrm{D}, \mathrm{E}$ and $\mathrm{F}$ contribute to the response functions at Portishead and St. Malo in the semi-diurnal tidal band. It shows the full response function and the background between 11 and $14 \mathrm{rad} \mathrm{day}^{-1}$ and the individual resonance contributions at 12 and $13 \mathrm{rad} \mathrm{day}^{-1}$.

At Portishead the main contribution to both the amplitude and curvature of the response function comes from resonance D. There are also significant contributions from resonances $\mathrm{E}$ and $\mathrm{F}$. Their phases do not change very much between 12 and $13 \mathrm{rad} \mathrm{day}^{-1}$ but they are important in that they significantly reduce the amplitude of the response function in the tidal band.

At St. Malo the contribution of resonance D is much smaller, but the large change in their relative phases means that resonances $\mathrm{D}$ and $\mathrm{E}$ act roughly in phase. This generates a response function amplitude that is roughly twice that which would be obtained by either resonance acting alone.

\footnotetext{
${ }^{1}$ Poles on the imaginary axis do not have a conjugate.
}

Resonance F has a very small amplitude at St. Malo, but resonance $\mathrm{C}$, which is negligible at Portishead, has a significant contribution here.

Although the resonance expansion works reasonably well in the semi-diurnal tidal band, it is much less successful in the diurnal band. The only one of the four locations of Fig. 4 where a useful result could be obtained was Workington, where resonances B plus resonance D leave an almost constant background term, approximately $20 \%$ of the full amplitude.

This failure is discussed in Appendix B. The main conclusions are that, first, in the diurnal band, the resonance $\mathrm{B}$ becomes strongly coupled to many more continental shelf and Rossby waves than is shown in Table 3. Secondly, although the background has a similar amplitude to that in the semidiurnal band, in the diurnal band its amplitude is much larger than the resonance contributions. Finally, the changes in the background over the diurnal band are comparable with the resonance contribution, and so strongly distort the resonance loop. As a result, any useful expansion needs to accurately represent the background as well as including many of the continental shelf/Rossby wave resonances.

\section{Discussion}

\subsection{The resonances}

The main result of this study is that the tides of the English Channel are dominated by two resonances associated with the Bristol Channel. They both involve quarter-wavelength standing waves between the Bristol Channel and the edge of the continental shelf, but in one the amplitude in the Gulf of St. Malo is weak and in the other it is of comparable amplitude. The phase relationship between the two modes also 


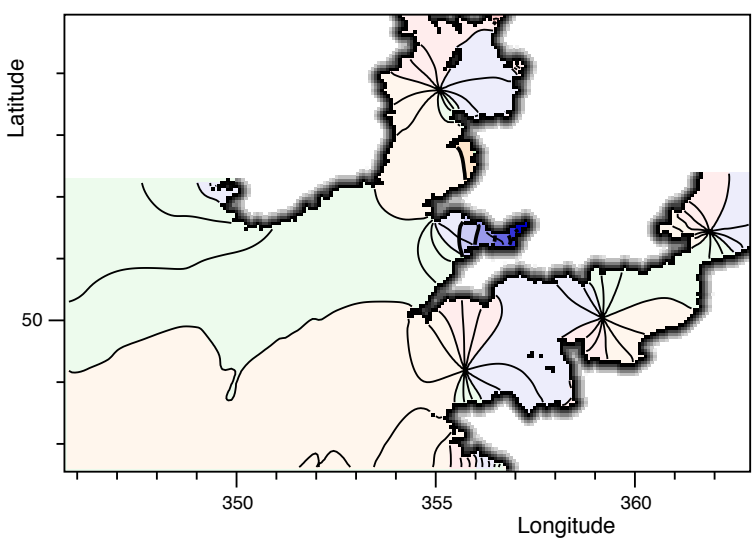

Resonance F

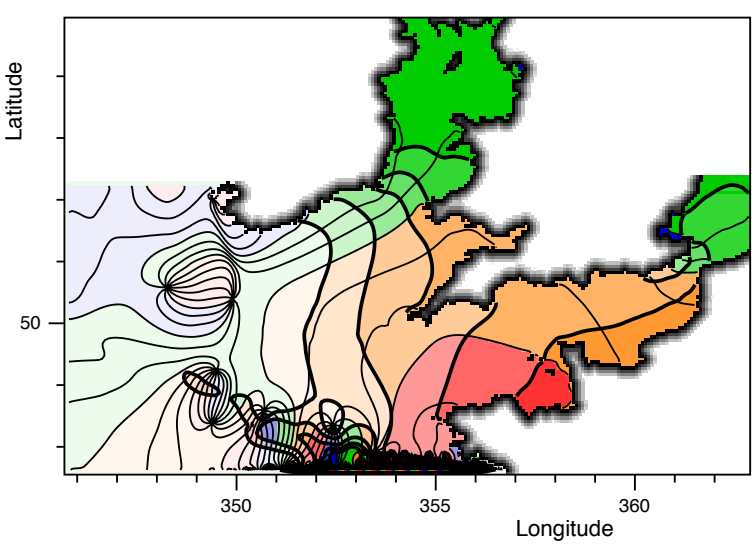

Resonance Bd

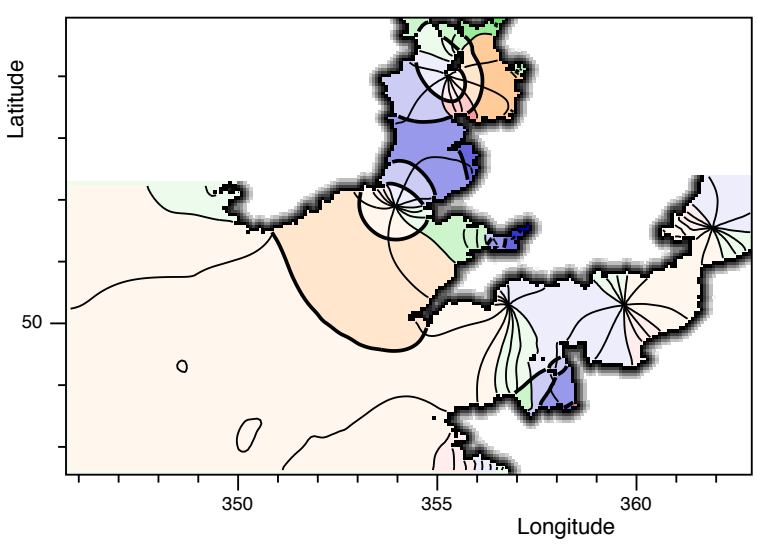

Resonance G

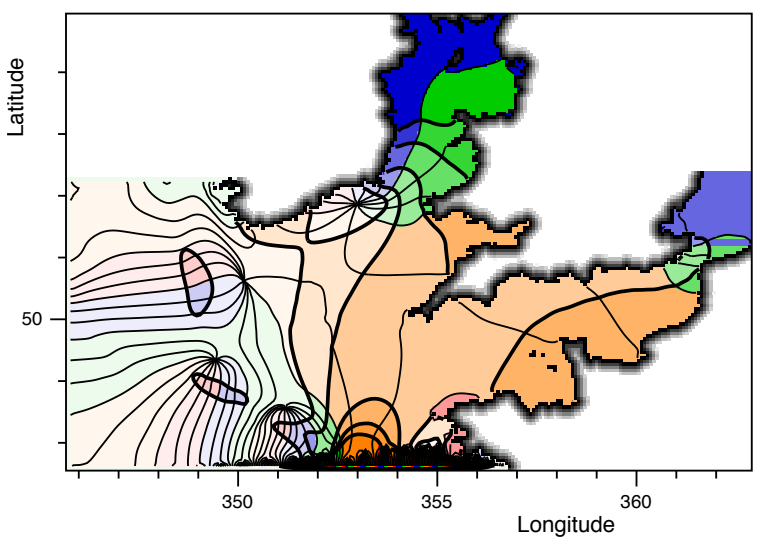

Resonance Bf

Fig. 9. Amplitude and phase of the resonances F, G, Bd and Bf (table 3). Colours as in Fig. 8. F and G normalized so that the maximum amplitude is 1. Bd and Bf normalized to show the small amplitude structure on the continental shelf.

differ so that they cancel each other in the Bristol Channel yet reinforce each other in the Gulf of St. Malo.

The model also shows that continental shelf waves have a significant effect on the diurnal tides throughout the region. As a result it is usually not possible to expand the response function in the diurnal band in terms of a few resonances plus a small background.

\subsection{Model limitations}

Although the above results appear to be robust, the model has a number of limitations which need to be kept in mind. Thus even with the limited area model used here, there appears to be a problem with resolution in the Bristol Channel. In both the M2 solution (Fig. 2) and the Bristol Channel modes (Fig. 8) there are large changes in amplitude in a region which is only poorly resolved by the model grid.

A previous study, which used half the present resolution, was found to underestimate the tides near the head of the channel. In contrast the present study gives amplitudes which are too high. Increasing resolution by a further factor of 2 was considered, but it would have increased the computational cost by a factor of 8 , and this was considered impractical for the present preliminary study. However further studies are needed with much better resolution in this region.

The present model also used closed boundaries in the southern part of the North Sea and in the North Channel of the Irish Sea. Tests were carried out with different positions for the boundary in the North Sea, and it was found that as long as the boundary was beyond the Thames Estuary, the effect on the tides of the English Channel was small. As a result except for resonance $\mathrm{C}$, which has significant amplitude beyond the Dover Strait, the effect of this boundary should be small. No similar tests have been made with the boundary in the North Channel, but there is no reason to expect the effect to be larger.

Finally, the model used an open boundary condition which does not allow for the energy trapped in the resonances to be lost to the rest of the ocean. Webb (2011) investigated this effect in a one-dimensional model and found that radiation had a only small effect on the real component of the resonance angular velocity but could double the imaginary component. 


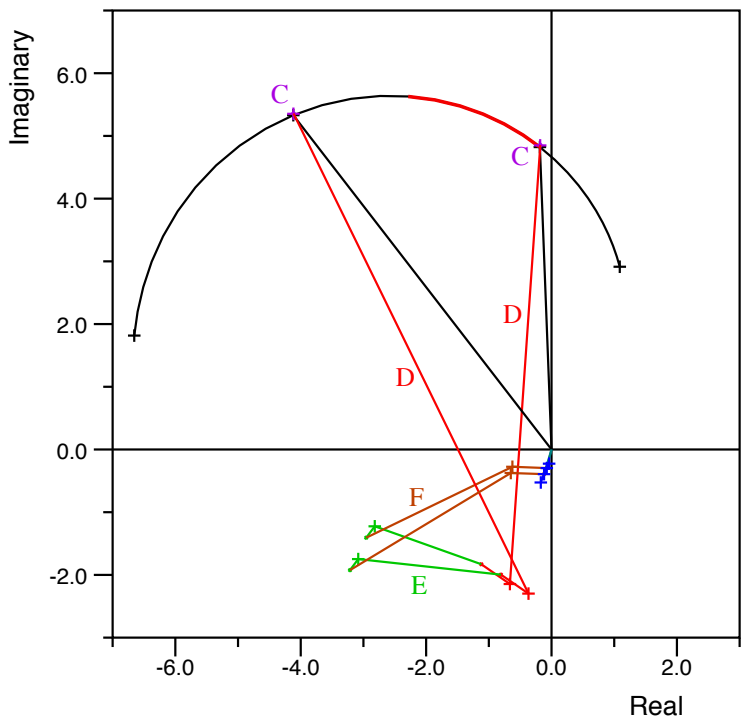

Portishead

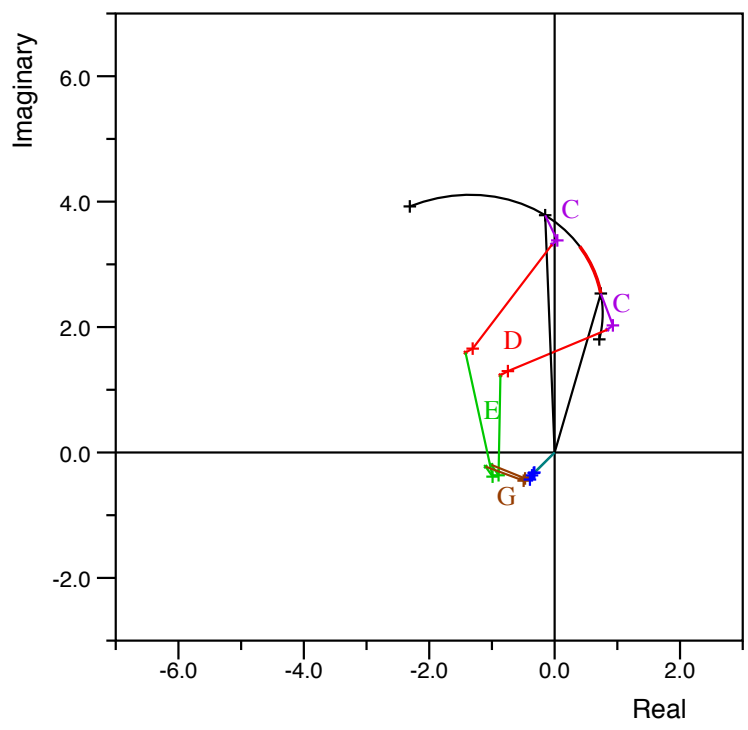

St Malo

Fig. 10. Polar plot of the Portishead and St. Malo response functions (black) between 11 and 14 radians per day. Crosses are at intervals of 1 radian per day. The figure also shows the contributions at 12 and 13 rad day $^{-1}$ of resonances $\mathrm{C}$ (purple), D (red), E (green), F (brown) and $\mathrm{G}$ (dark brown) of Table 3, their much smaller conjugates (same colours) and the residual (blue).

If this is also true for the resonances reported here, it would double their distance from the real axis.

However a later study used the present model with a radiational boundary condition based on Flather (1976). This resulted in much smaller increases in the distance of the resonances from the real axis. The results are discussed in Webb (2013) and it is possible that the small increase results from reflection of the outgoing wave at the open boundary. This could be due to the Coriolis term or the direction of propagation of the outgoing wave.

In either case the spatial structure of each mode will also change. If the change is relatively small and we assume the one-dimensional result is correct, the maximum contribution of each pole to points along the real axis will be approximately halved. This will smooth the response at real values of angular velocity and may increase the relative importance of the background.

\subsection{A Bristol Channel barrage}

The Bristol Channel resonances are of particular interest because of the potential use of the tides there for generating electricity. Table 3 shows that the key resonances have real components of angular velocity equal to 13.14 and 14.66 radians per day, which are just above the range of the semidiurnal tides. If a barrage is built in the Bristol Channel, then the effective shelf width will be reduced, and this would move the resonances to a higher angular velocity, away from the tidal band.
This may result in a reduction of the amount of tidal energy that could be extracted using the barrage. The coupling between the semi-diurnal tides of the Bristol Channel and the Gulf of St. Malo also means that a barrage in the Bristol Channel is likely to change the tides near St. Malo and so affect the performance of the Rance tidal power station.

\section{Appendix A}

\section{The Coriolis term}

For the Coriolis terms of Eq. (6), the value of $(f v)$ at $u_{i, j}$ is often calculated as a simple average over neighbouring $v$ points, but Espelid et al. (2000) show that this approximation does not conserve energy. Earlier, Arakawa and Lamb (1981) proposed an energy-conserving scheme in which both the kinetic energy and the Coriolis term $f$ were defined for "vorticity" cells. These cells are centred on the corner points of the cell of Fig. 1 for which the velocity in each direction is defined as the average of the two closest model velocity cells. A similar scheme is used in the Nemo model (Madec, 2008).

For models which do not use a finite-difference scheme based on vorticity, Espelid et al. (2000) propose a simpler scheme which removes the averaging. Their scheme is similar to the one used for the present model (Eq. A1) but they place all Coriolis terms, $f$, inside the summation and, for each pair, replace them by their geometric average. 


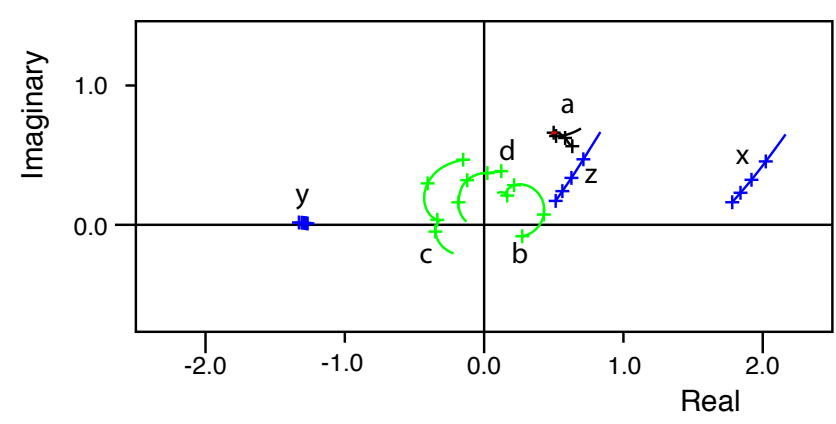

Fig. A1. Components of response function at Portishead between 4 and $8 \mathrm{rad} \mathrm{day}^{-1}$. Crosses at 4, 5, 6 and $7 \mathrm{rad} /$ day. (a) Full response function with diurnal tidal band in red, (b) resonances B of Table 3, (c) remaining shelf and Rossby resonances, (d) sum of (b) and (c), (x) gravity wave resonances of Table 3 excluding $\mathrm{B}$, (y) waves with real component of angular velocity between 30 and $100 \mathrm{rad} \mathrm{day}^{-1}$, (z) sum of (x) and (y). The sum of (d) and (z) is not plotted but at this resolution it would overlay (a).

For the present model, energy conservation is achieved by expanding the term $(f v)_{u ; i, j}$ of Eq. (6) as

$$
\begin{aligned}
(f v)_{u ; i, j} & =f_{u ; i, j} \sum_{i^{\prime}, j^{\prime}} v_{i^{\prime}, j^{\prime}} C\left(i, j ; i^{\prime}, j^{\prime}\right) / 4, \\
C\left(i, j ; i^{\prime}, j^{\prime}\right) & =\frac{2 f_{v ; i^{\prime}, j^{\prime}} V_{v ; i^{\prime}, j^{\prime}}}{\left(f_{u ; i, j} V_{u ; i, j}+f_{v ; i^{\prime}, j^{\prime}} V_{v ; i^{\prime}, j^{\prime}}\right)} .
\end{aligned}
$$

The sum $\left(i^{\prime}, j^{\prime}\right)$ is over the four $v$ grid points closest to each $u$ grid point, and $V_{u ; i^{\prime}, j^{\prime}}$ and $V_{v ; i^{\prime}, j^{\prime}}$ are the volumes of the grid cells surrounding the $u$ and $v$ points. The term $(f u)_{v ; i, j}$ is treated in a similar manner.

\section{Appendix B}

\section{The resonance expansion}

The difficulty in finding a simple resonance fit to the diurnal response function at Portishead prompted a check that the resonance expansion (Eq. 12) was valid and that the background term eventually tended to zero as more resonances were added. The check was carried out by first identifying as many resonances as possible in the regions covered by Figs. 5 to 7 .

To do this the raw data used to generate the figures was scanned to find all local maxima. The maxima were then used as starting points for the iterative method used to find resonance eigenvalues and eigenfunctions. This scheme produced a total of 541 resonances. The results were checked by subtracting the resulting resonance response function from the original raw data. The resulting figure showed three welldefined peaks, an almost constant background term and an area of very small-scale structure close to the origin.

The three peaks were found to be additional resonances. When these are added, the response function in the diurnal tidal band has the right shape, but it shows an almost constant offset from the model results.

As the offset could to be due to shorter wavelength gravity waves outside the range covered, the analysis was extended to include all of the region enclosed by the origin and $(0-10 i),(100-10 i)$ and $100 \mathrm{rad} \mathrm{day}^{-1}$. This gave a total of 627 resonances and removed most of the remaining error, the absolute error at $6 \mathrm{rad} \mathrm{day}^{-1}$ being reduced to less than 0.008 and that at the origin to less than 0.02 . These errors are likely to be due to the contributions from further short-wavelength modes; that is, gravity waves at even higher angular velocities and the shelf or Rossby wave modes that generate the noise near the origin.

From these results it was deduced that the basic resonance expansion was valid and the problem with the diurnal tides comes from the mixing of a shelf quarter-wavelength gravity wave response with continental shelf waves with similar complex angular velocities.

These different contributions to the diurnal band response function are illustrated in Fig. A1. It shows the contribution of the resonances $\mathrm{B}$ of Table 3, the contribution of the remaining continental shelf/Rossby waves (which still contains a resonant kink) and the sum, which shows a small resonant loop. It also shows the rapidly varying contribution from the other resonances of Table 3 and the almost constant contribution from resonances between 30 and $100 \mathrm{rad} \mathrm{day}^{-1}$.

A study of the individual terms in the resonance expansion emphasized the importance of cancellation effects. At $6 \mathrm{rad} \mathrm{day}^{-1}$ the amplitude of the response function at Portishead is 0.83 . When the resonance contributions are listed in order, six gravity wave resonances have amplitudes greater than 0.8 . There are 30 resonances with amplitudes greater than 0.1 , showing a roughly equal split between gravity and shelf/Rossby waves, and 85 with amplitudes greater than 0.01 . The pattern then continues with roughly equal numbers of the two classes waves, the error of the resonance expansion only reducing to less than 0.1 after 108 resonances.

Acknowledgements. I wish to thank the two reviewers for their detailed comments and the resulting on-line discussion and improvements to the paper. Thanks also to the editor, Neil Wells. He wishes me to point out that the on-line discussion covers many issues which workers in the field may not be fully aware. Finally thanks to the Marine Systems Modelling Group at NOC who funded publication of this paper and provided other crucial support for the study.

Edited by: N. Wells

\section{References}

Arakawa, A.: Computational design for long-term numerical integration of the equations of fluid motion: Two-dimensional incompressible flow, J. Computat. Phys., 1, 119-143, 1966. 
Arakawa, A. and Lamb, V. R.: A Potential Enstrophy and Energy Conserving Scheme for the Shallow Water Equations, Monthly Weather Rev., 109, 19-36, 1981.

Arbic, B. K., St-Laurent, P., Sutherland, G., and Garrett, C.: On the resonance and influence of the tides in Ungava Bay and Hudson Strait, Geophys. Res. Lett, 34, L17606, ,doi:10.1029/2007GL030845, 2007.

Arbic, B. K., Karsten, R. H., and Garrett, C.: On Tidal Resonance in the Global Ocean and the Back-Effect of Coastal Tides upon Open-Ocean Tides, Atmos.-Oc., 47, 239-266, 2009.

Brink, K.: Coastal-trapped Waves and Wind-driven Currents over the Continental Shelf, Ann. Rev. Fluid Mechan., 23, 389-412, 1991.

Buchwald, V. T.: Resonance of Poincaré waves on a continental shelf, Austr. J. Mar. Freshwater Res., 31, 451-457, 1980.

Cartwright, D. E., Edden, A. C., Spencer, R., and Vassey, J. M.: The tides of the northeast Atlantic Ocean, Philos. T. R. Soc. Lond., 298, 87-139, 1980a.

Cartwright, D. E., Huthnance, J., Spencer, R., and Vassey, J. M.: On the St Kilda shelf tidal regime, Deep-Sea Res., 27A, 61-70, 1980b.

Clarke, A. J. and Battisti, D. S.: The effect of continental shelves on tides, Deep-Sea Res., 28A, 665-682, 1981.

Egbert, G. and Ray, R.: Significant dissipation of tidal energy in the deep ocean inferred from satellite altimeter data, Nature, 405, 775-778, 2000.

Espelid, T. O., Berntsen, J., and Barthel, K.: Conservation of energy for schemes applied to the propagation of shallow-water inertiagravity waves in regions with varying depth, Int. J. Numer. Engng., 1521-1545, 2000.

Flather, R. A.: A Tidal Model of the North-west European Continental Shelf, Memoires Societé Royale des Sciences de Lige, 10, 141-164, 1976

Flather, R. A.: Estimates of extreme conditions of tide and surge using a numerical model of the north-west European continental shelf, Estuarine, Coast. Shelf Sci., 24, 69-93, 1987.

Fong, S. and Heaps, N.: Note on the quarter-wave tidal resonance in the Bristol Channnel, Institute of Oceanographic Sciences, Report No., 63, 15, 1978.

Grignon, L.: Tidal resonances on the North-West European Shelf, Master's thesis, University of Southampton, School of Ocean and Earth Science, 2005

Heath, R.: Resonant period and Q of the Celtic Sea and Bristol Channel, Estuarine, Coast. Shelf Sci., 12, 291-301, 1981.

Huthnance, J. M.: On Trapped Waves over a Continental Shelf, J. Fluid Mechan., 69, 689-704, 1975.

Huthnance, J. M.: On shelf-sea resonance with application to Brazilian M3 tides, Deep-Sea Res., 27A, 347-366, 1980.
IOC, IHO, and BODC: Centenary Edition of the GEBCO Digital Atlas, published on CD-ROM on behalf of the Intergovernmental Oceanographic Commission and the International Hydrographic Organization as part of the General Bathymetric Chart of the Oceans, British Oceanographic Data Centre, Liverpool, UK, 2003.

Jones, J. E. and Davies, A. M.: An intercomparison between finite difference and finite element (TELEMAC) approaches to modelling west coast of Britain tides, Ocean Dynam., 55, 178-198, 2005.

Kwong, S. C., Davies, A. M., and Flather, R. A.: A threedimensional model of the principal tides on the European shelf, Prog. Oceanogr., 39, 205-262, 1997.

Le Provost, C., Rougier, G., and Poncet, A.: Numerical Modeling of the Harmonic Constituents of the Tides, with Application to the English Channel, J. Phys. Oceanogr., 11, 1123-1138, 1981.

Madec, G.: NEMO Ocean Engine, Note du Pole de modélisation, No. 27, Institut Pierre-Simon Laplace (IPSL), France, http:// www.nemo-ocean.eu/About-NEMO/Reference-manuals, 2008.

Owen, A.: A Three-Dimensional Model of the Bristol Channel, J. Phys. Oceanogr., 10, 1290 - 1302, 1980.

Pingree, R. D. and Griffiths, D. K.: Tidal fronts on the shelf seas around the British Isles, J. Geophys. Res., 83, 4615-4622, 1978.

Sinha, B. and Pingree, R.: The principal lunar semidiurnal tide and its harmonics: baseline solutions for M2 and M4 constituents on the North-West European Continental Shelf, Contin. Shelf Res., 17, 1321-1365, 1997.

Stephens, C. V.: A three-dimensional model for tides and salinity in the Bristol Channel, Contin. Shelf Res., 6, 531-560, 1986.

Uncles, R.: Physical properties and processes in the Bristol Channel and Severn Estuary, Mar. Pollut. Bull., 61, 5-20, 2010.

Webb, D. J.: Green's Function and Tidal Prediction, Rev. Geophys. Space Phys., 12, 103-116, 1973.

Webb, D. J.: A Model of Continental Shelf Resonances, Deep-Sea Res., 23, 1-15, 1976.

Webb, D. J.: Numerical Model of the Tides in the Gulf of Carpentaria and Arafura Sea, Austr. J. Mar. Freshwater Res., 32, 31-44, 1981.

Webb, D. J.: Notes on a 1-D Model of Continental Shelf Resonances, Research and Consultancy Report 85, National Oceanography Centre, Southampton, http://eprints.soton.ac.uk/171197, 2011.

Webb, D. J.: On the Shelf Resonances of the Gulf of Carpentaria and the Arafura Sea, Ocean Science, 8, 733-750, doi:10.5194/os-8733-2012, 2012.

Webb, D. J.: On the Impact of a Radiational Open Boundary Condition on Continental Shelf Resonances, National Oceanography Centre, Internal Document 06, National Oceanography Centre, Southampton, http://eprints.soton.ac.uk/349401, 2013 